\title{
SHORT COMMUNICATION \\ What is said or how it is said makes a difference: role of the right fronto-parietal operculum in emotional prosody as revealed by repetitive TMS
}

\author{
Sophie van Rijn, ${ }^{1,2}$ André Aleman, ${ }^{1,3}$ Eric van Diessen, ${ }^{2}$ Celine Berckmoes, ${ }^{4}$ Guy Vingerhoets ${ }^{4}$ and René S. Kahn ${ }^{2}$ \\ ${ }^{1}$ Helmholtz Instituut, Psychological Laboratory, Universiteit Utrecht, PO Box 80125, 3508 TC Utrecht, the Netherlands \\ ${ }^{2}$ Department of Psychiatry, Rudolf Magnus Institute for Neuroscience, University Medical Centre Utrecht, Heidelberglaan 100, \\ 3508 GA Utrecht, the Netherlands, \\ ${ }^{3} \mathrm{BCN}$ Neurolmaging Centre, University of Groningen, Groningen, the Netherlands \\ ${ }^{4}$ Laboratory for Neuropsychology, Ghent University, Ghent, Belgium
}

Keywords: emotion, prosody, somatosensory cortex, TMS, withdrawal-approach

\begin{abstract}
Emotional signals in spoken language can be conveyed by semantic as well as prosodic cues. We investigated the role of the frontoparietal operculum, a somatosensory area where the lips, tongue and jaw are represented, in the right hemisphere to detection of emotion in prosody vs. semantics. A total of 14 healthy volunteers participated in the present experiment, which involved transcranial magnetic stimulation (TMS) in combination with frameless stereotaxy. As predicted, compared with sham stimulation, TMS over the right fronto-parietal operculum differentially affected the reaction times for detection of emotional prosody vs. emotional semantics, showing that there is a dissociation at a neuroanatomical level. Detection of withdrawal emotions (fear and sadness) in prosody was delayed significantly by TMS. No effects of TMS were observed for approach emotions (happiness and anger). We propose that the right fronto-parietal operculum is not globally involved in emotion evaluation, but sensitive to specific forms of emotional discrimination and emotion types.
\end{abstract}

\section{Introduction}

In order to understand messages in verbal communication, it is important to pay attention not only to what is said but also to how it is said. Besides the semantic (linguistic) meaning of words, features such as intonation and loudness in speech can also convey crucial information. The term prosody has been introduced to describe these nonsemantic cues in spoken language. Prosodic cues can have linguistic as well as affective functions. Linguistic functions are, for example, emphasizing important parts of the message or presenting information as a statement or a question. Affective functions of prosody are also important for understanding intentions of others, because variations in tone of voice, such as intonation and loudness, provide information about the emotional state of the speaker. Thus, the emotional relevance of a spoken message may be not only be conveyed by meaning of words (i.e. emotional semantics), but also expressed in emotional prosody. The present study is focused on processing of affective information conveyed by these different aspects of spoken language, i.e. emotional semantics vs. emotional prosody.

These two different communicative channels of information about the emotional relevance of a spoken message, i.e. semantic and prosodic, could be dissociable at a neuroanatomical level. A range

Correspondence: Dr S. van Rijn, as above.

E-mail: s.vanrijn@fss.uu.nl

Received 15 November 2004, revised 23 March 2005, accepted 28 March 2005 of studies have suggested that the left hemisphere seems to be specialized for most aspects of language processing, including linguistic functions of prosody (e.g. stress evaluation; Aleman et al., 2004). However, the right hemisphere (RH) appears to be involved in nonlinguistic components, such as emotional prosody (Baum \& Pell, 1999).

The best evidence regarding RH involvement in affective prosody comes from studies directly contrasting emotional semantics with emotional prosody. In contrast to processing of emotional semantics, emotional prosody involves the right hemisphere as is shown in imaging studies (Baum \& Pell, 1999; Mitchell et al., 2003; Vingerhoets et al., 2003). These findings suggest that the role of the right hemisphere in emotional prosody is not a specialization in emotion detection in general, but that RH involvement depends on the communication channel through which emotional relevance is presented.

In addition to the type of communication channel, involvement of the right hemisphere might also depend on the type of emotion. A widely used categorization of emotions is based on broader constructs of behavioural inhibition and activation (Sutton \& Davidson, 1997). Whereas withdrawal emotions such as fear and sadness are accompanied by inhibitory motivational tendencies, approach emotions, such as anger and happiness, are accompanied by activational motivational tendencies. Right hemisphere-damaged patients show specific deficits in processing withdrawal emotions, i.e. fear and sadness, in contrast to normal processing of approach emotions such 
as happiness and anger (Mandal et al., 1999; Anderson et al., 2000). Also, it has been proposed that whereas the left frontal cortical region is important for expression and experience of approach emotions, the right frontal cortical region is involved in expression and experience of withdrawal emotions (Harmon-Jones, 2003; Sutton \& Davidson, 1997).

Recently, Vingerhoets et al. (2003) have measured blood flow velocity (BFV) with functional transcranial Doppler ultrasonography (fTCD) to study the contribution of the right and left hemisphere to the detection of emotion in prosody vs. detection of emotion in semantics of spoken language. fTDC is a noninvasive tool with high temporal resolution that allows a continuous monitoring of blood flow velocity in the basal cerebral arteries, thereby reflecting changes in cerebral metabolism that accompanies mental activity. During detection of emotion in semantics a significant left-hemispheric lateralization of $\mathrm{BFV}$ was observed. This lateralization effect disappeared when attention was shifted to discriminating emotion in prosody, due to a rise in right hemispheric BFV. Although metabolism in the right hemisphere is correlated with detection of emotional prosody in this study, the question remains whether the $\mathrm{RH}$ is causally involved in emotional prosody. In addition, is not clear which brain region within the $\mathrm{RH}$ is critically involved in emotional prosody discrimination and whether such a region is sensitive to specific types of emotions.

As an extension of the study of Vingerhoets et al. (2003), we used transcranial magnetic stimulation (TMS) to answer these questions. TMS delivers short magnetic pulses that penetrate the skull and disrupt neural processing in a noninvasive, reversible way (Walsh \& PascualLeone, 2003). This virtual lesion technique allows investigation of the causal relation between neural activity and performance at a behavioural level. In addition, specific brain regions can be targeted, depending on the type of coil used for stimulation. Ideally, such regions should be determined on the basis of lesion data in human patients. Recently, a comprehensive three-dimensional (3-D) lesion study investigating the neural correlates of emotional prosody has been published (Adolphs et al., 2002). In a 3-D reconstruction of all the lesions in focal brain-damaged patients, the density of lesionoverlap was analysed as a function of task performance. The most robust finding was a relation between damage to the right frontoparietal operculum and compromised detection of emotion from prosody.

Our prediction was that, when compared with sham stimulation, TMS over the right fronto-parietal operculum differentially affects reaction times for detection of emotional prosody when compared to emotional semantics, showing that: (i) distinct neuroanatomical networks underlie attention to emotional prosody vs. emotional semantics; and (ii), the right fronto-parietal operculum is sensitive to specific forms of emotional discrimination (semantic/prosodic) and emotion types (withdrawal/approach emotions), rather then globally involved in emotion evaluation.

\section{Materials and methods \\ Subjects}

Fourteen healthy adult subjects participated in the study after giving written informed consent (19-27 years, mean age 23 years; 8 males/ 6 females). Handedness was measured with the Edinburgh Handedness Inventory $(-24=$ exclusively left handed; $0=$ no preference; 24 = exclusively right handed; Oldfield, 1971). All participants were right-handed (mean 21.3, SD = 2.6). Subjects were screened for contraindications to TMS, neurological and medical problems. The experiment was conducted in accordance to the Declaration of Helsinki and local ethics board approval (University Medical Centre Utrecht).

\section{Apparatus}

For TMS, we used a MagStim Rapid magnetic stimulator (MagStim Co, Whitland, UK) with a figure-of-eight magnetic coil with a diameter of $70 \mathrm{~mm}$ for each loop.

\section{Experimental protocol}

The offline TMS experiment comprised two conditions, TMS and sham, each followed by two tasks - emotional semantics and emotional prosody. The order of both the conditions (stimulation over the right and left fronto-parietal operculum for real TMS and sham, respectively) and the tasks was counterbalanced over subjects. The minimum time interval between TMS and sham was $30 \mathrm{~min}$ to prevent carry-over effects (cf. Kosslyn et al., 1999; Oliveri et al., 2004).

\section{TMS over the fronto-parietal operculum}

First, the motor threshold (MT) was determined for each subject, defined as the lowest stimulation intensity that induced visible finger movements in at least 5 out of 10 trials when TMS was applied to the motor cortex (Pridmore et al., 1998).

Second, localization of the right fronto-parietal operculum was accomplished individually using structural magnetic resonance imaging (MRI) and neuronavigation by frameless stereotaxy $(\mathrm{NeNa}$; Neggers et al., 2004). Anatomical T1-weighted MRI scans were used to delineate the fronto-parietal operculum bilaterally in each participant. The fronto-parietal operculum was defined as the inferior pericentral sulcus area (see Fig. 1) and was delineated independently by two separate raters as a region of interest (ROI) of approximately 1$2 \mathrm{~cm}$ in diameter, compatable with the size of the cortical region that TMS typically affects (Walsh \& Cowey, 2000). The size and location of the ROI was based on the regions with highest lesion-overlap values of the fronto-parietal operculum from the 3-D lesion study by Adolphs et al. (2002).

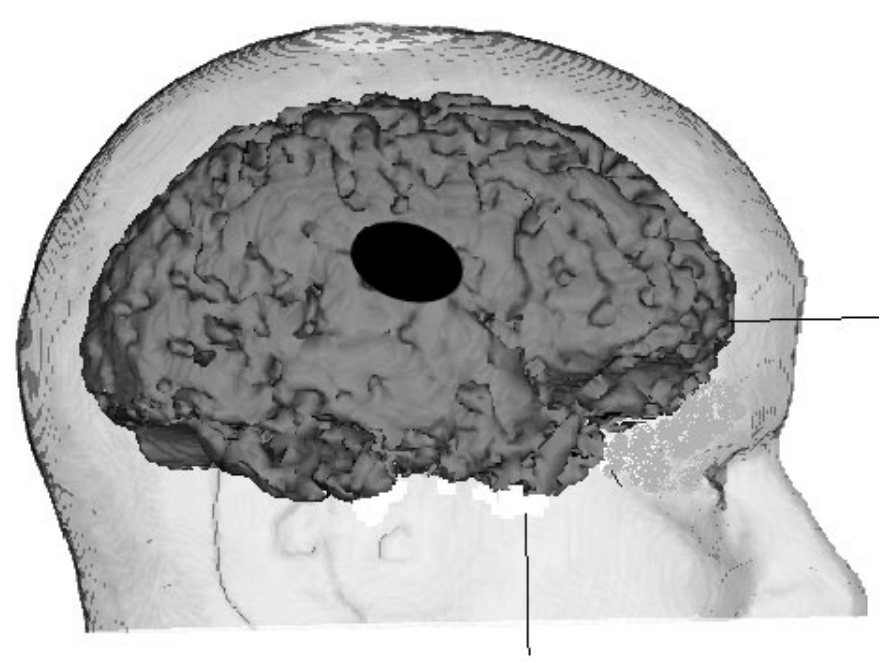

FIG. 1. The fronto-parietal operculum (in black) as a region of interest (ROI), based on anatomical T1-weighted MRI images, projected on a rendering of an individual brain. 
The structural MRI scan as well as the ROI maps, representing the fronto-parietal operculum bilaterally, were loaded into $\mathrm{NeNa}$ before the experiment. Six anatomical landmarks were set on the skin rendering. The participant would then be seated, the head fixed in a head support, and a rubber head cap placed over the head. The positions of the same anatomical landmarks were measured with the MiniBIRD position-tracker probe, directly at the head of the participant, and the mapping between 3-D space and MRI space was calculated. After coregistration, the fronto-parietal operculum was targeted by 'looking' through the $\mathrm{NeNa}$ probe to the renderings of the skin and ROI on the screen and the position directly overlying the ROI was marked on the head-cap (for more details on localization using the NeNa system, see Neggers et al., 2004).

In the stimulation condition, subjects were then stimulated with $1 \mathrm{~Hz}$ TMS for $12 \mathrm{~min}$ over the right fronto-parietal operculum, marked at the head-cap, at $90 \%$ of the MT. These parameters have been applied in earlier studies of cognitive TMS (for an overview see Robertson et al., 2003) and have been shown to affect brain metabolism (Mottaghy et al., 2003). The coil was held manually with the handle pointing backwards and kept tangential to the subject's scalp.

In the control condition, sham stimulation was applied for $12 \mathrm{~min}$ over the left fronto-parietal operculum by rotating the coil $90^{\circ}$, in order to direct the magnetic field away from the brain, but controlling for the characteristic 'click' and sensation on the scalp. Because concerns have been raised that this sham method could still affect brain activity (when rotated $45^{\circ}$; Loo et al., 2000), we stimulated at a lower intensity of $35 \%$ of stimulator output in the sham condition, and over the left hemisphere in order to avoid weakening of the manipulation, resulting in a null effect.

\section{Emotion discrimination tasks}

Immediately after (sham) stimulation, subjects were required to identify the emotion conveyed by prosody or semantics of a number of sentences. We used the tasks designed by Vingerhoets et al. (2003). Each task (prosody and semantics) was assessed twice (after TMS and after sham stimulation), with a different list of stimuli. Each task presentation was preceded by four practice sentences. The order of the tasks was counterbalanced over subjects.

Of the 24 sentences in each task, six were happy, six were sad, six were angry and six were fearful. Sentences were of approximately equal length and were articulated by two professional actors, one male and one female. The digitized stimuli were presented binaurally through earphones. During listening, the emotions to be discriminated were presented on the computer screen. In the prosody task, affective discrimination was based on the affective tone of voice. In this task, the content of the sentences was not affective (i.e. always neutral). In the semantic task, affective discrimination was based on the semantic content of the sentences. In this task, tone of voice was not affective (ie. always neutral). As soon as they identified the emotion expressed in the sentence, either based on content or tone of voice, subjects were required to pronounce that particular emotion in a microphone that was connected to the computer. Their responses, both selected emotion and reaction time for detection, were collected. The total duration for completing one of either tasks was $7 \mathrm{~min}$.

\section{Statistical analyses}

Statistical analyses were performed using Statistical Package for the Social Sciences 11.5.0 (2002). Accuracy and reaction times for detection of emotion in semantics and in prosody were chosen as dependent variables. Reaction times for incorrect responses were excluded from analyses. $2 \times 2$ GLM repeated measures analyses with TMS (stimulation, sham) and Task (prosody, semantics) as independent factors were used to test the effects of TMS on the two tasks. Post $h o c$ analyses were performed using paired $t$-tests. $P$-values were set at 0.05 , two-tailed.

\section{Results}

The mean MT was $49.7 \%$ ( $\mathrm{SD}=3.7 \%$ ) of stimulator output.

\section{Accuracy}

No significant TMS by Task interaction was observed for accuracy in detection of emotion in all four conditions $\left(F_{1,13}=0.42, P=0.53\right)$. At baseline (sham condition), participants were significantly more accurate in identifying emotion in semantics compared to prosody $\left(t_{1,13}=2.98, P=0.01\right)$.

Regarding the different types of emotions, again no TMS by Task interaction was observed for withdrawal emotions, i.e. fearful and sad $\left(F_{1,13}=0.11, P=0.74\right)$ and approach emotions, i.e. anger and happy $\left(F_{1,13}=0.92, P=0.35\right)$. At baseline (sham condition), paired $t$-tests showed that participants were significantly more accurate at identifying approach emotions when compared to withdrawal emotions in both the prosody task $\left(t_{1,13}=4.74, P=0.001\right)$ and the semantics task $\left(t_{1,13}=3.48, P=0.004\right)$, which is in accordance with other studies. Accuracy scores are presented in Table 1 .

\section{Reaction times}

Only reaction times for correct responses were included in the analyses.

First, we explored TMS effects on emotional prosody vs. emotional semantics when all emotions (happy, sad, anger, fear) were taken together. As expected, reaction times for detection of emotion conveyed by prosody were shorter when compared to emotion in semantics $(t=-3.7, P=0.002)$. Emotion in tone of voice can be detected well before completion of the sentence, whereas critical words can be positioned at the end of a sentence when relying on semantic information.

Regarding reaction times for detection of emotion in all four conditions, a significant TMS by Task interaction was present $\left(F_{1,13}=9.31, P=0.009\right)$. Post hoc tests failed to reach significance ( $P=0.32$ and $P=0.06$, for prosody and semantics, respectively), which implies that the effect is driven by a combination of both a decrease in semantic reaction times and an increase in prosody reaction times.

In the crucial analysis, the four types of emotions were grouped into two different categories; withdrawal (fearful and sad) vs. approach

TABle 1. Percentage emotions identified correctly in all conditions

\begin{tabular}{llll}
\hline & \multicolumn{3}{l}{ Emotions identified correctly (\%) } \\
\cline { 2 - 4 } & All emotions & Withdrawal emotions & Approach emotions \\
\hline Sham prosody & $84.2 \pm 8.2$ & $73.2 \pm 15.4$ & $94.6 \pm 5.3$ \\
TMS prosody & $81.5 \pm 9.7$ & $70.8 \pm 14.5$ & $92.3 \pm 7.6$ \\
Sham semantics & $93.7 \pm 4.8$ & $89.9 \pm 8.1$ & $97.6 \pm 3.9$ \\
TMS semantics & $94.0 \pm 4.8$ & $89.8 \pm 7.4$ & $98.2 \pm 3.5$ \\
\hline
\end{tabular}

Data are presented as mean $\pm \mathrm{SD}$. 


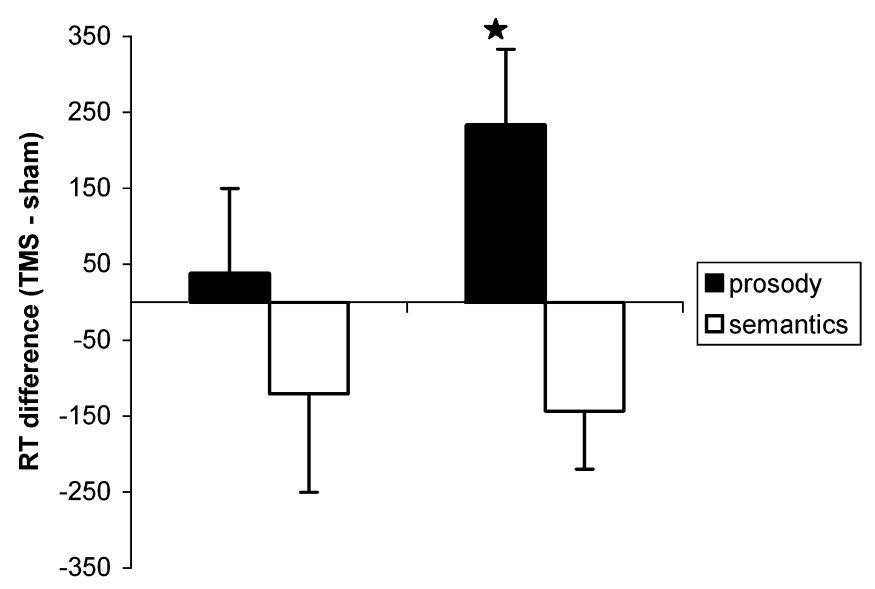

approach withdrawal

FIG. 2. TMS effects on detection of approach emotions (happy, anger) and withdrawal emotions (fear, sad) in prosody vs. semantics (mean $\pm \mathrm{SEM}$, $\left.t_{1,13}=2.34, P=0.036\right)$.

(anger and happy). For withdrawal emotions, again a significant TMS by Task interaction appeared $\left(F_{1,13}=9.13, P=0.01\right)$. Post hoc tests revealed a significant effect of TMS on reaction times for detection of withdrawal emotions in the prosody condition $\left(t_{1,13}=2.34\right.$, $P=0.03)$. The mean reaction times increased from $3554 \mathrm{~ms}$ $(\mathrm{SD}=576)$ at sham to $3788 \mathrm{~ms}(\mathrm{SD}=813)$ after TMS. In the semantics condition, the effects of TMS on reaction times for detection of withdrawal emotions did not reach significance $\left(t_{1,13}=-1.87\right.$, $P=0.08)$. In this condition the mean reaction times decreased from $3808 \mathrm{~ms}(\mathrm{SD}=356)$ at sham to $3664 \mathrm{~ms}(\mathrm{SD}=344)$ after TMS.

Interestingly, for approach emotions, happy and anger, no significant interaction between TMS and Task was observed $(P=0.26)$. Results are presented in Fig. 2.

\section{Discussion}

The present study used TMS to investigate the contribution of the fronto-parietal operculum in the RH to discrimination of emotions from prosodic and semantic information in spoken language. As predicted, TMS over the right fronto-parietal operculum differentially affected reaction times for detection of emotional prosody when compared to emotional semantics, indicating that at least partly separable neural networks are involved. Specifically, in the prosody condition detection of withdrawal emotions (fear and sadness), but not approach emotions (happiness and anger), was delayed by TMS over the right fronto-parietal operculum, suggesting that this region is crucial for detection of withdrawal emotions in prosody.

The neuroanatomical dissociation as revealed by the present study, is consistent with lesion, and neuroimaging studies, indicating that processing of emotional information from prosody and semantics may be differentially localized in the brain (Baum \& Pell, 1999; Mitchell et al., 2003; Vingerhoets et al., 2003). Whereas these studies are in essence correlational in nature, TMS allows a causal interpretation of the data. By using TMS in combination with frameless stereotaxy, we were not only able to attribute the changes in performance to disruptions in neural processing caused by TMS, but also link performance to a specific, well defined area in the brain. As shown in the present study, the fronto-parietal operculum in the right hemisphere appears to be part of a neural network selectively involved in detection of emotion, depending both on emotion type (i.e. withdrawal emotions) and whether information is conveyed by semantics or prosody.

The present evidence that emotion perception might be mediated by different neural substrates, depending on the communicative channel through which it is presented, is at odds with the 'right hemisphere dominance hypothesis', postulating that the right hemisphere is specialized for emotion evaluation, regardless of processing mode, such as lexical or prosodic (Blonder et al., 1991; Borod et al., 1993). It has been argued that right somatosensory areas are important understanding emotions of others and contribute to recognizing emotions by creating somatosensory representations based on internal simulation (Damasio, 1994; Adolphs et al., 2000). The fronto-parietal operculum forms part of the secondary somatosensory cortex where the lips, jaw and tongue are represented. Although speculative, specific knowledge of the emotional state of a speaker may be retrieved by reactivating a neural pattern in the fronto-parietal operculum that simulates emotion expressed in nonverbal cues in spoken language.

More specifically, it has been proposed that the somatosensory cortices may be an 'affective convergence zone', where emotion representations are formed not only irrespective of modality, but also aspecific for discrete emotions (Anderson \& Phelps, 2000). Findings from a recent study of patients with localized brain lesions shows that the somatosensory cortices are critical for understanding a broad range of emotional states from facial expressions (Adolphs et al., 2000). However, the present study shows specific effects for different emotion categories, suggesting that involvement of somatosensory areas may depend on specific emotion types. Indeed, there is evidence from studies with right hemisphere damaged patients who show specific deficits in detection of withdrawal emotions on faces, such as fear and sadness, in contrast to normal processing of approach emotions (Mandal et al., 1999; Anderson et al., 2000). A very recent TMS study has measured the effects of TMS over the right somatosensory cortex on perception of facial expressions of fear (withdrawal emotion) and happiness (approach emotion; Pourtois et al., 2004). Interestingly, only perception of fear, and not happiness, was disrupted by TMS over the right somatosensory cortex. Our findings are in line with this study, supporting the hypothesis that right somatosensory areas are important for perception of withdrawal emotions both in facial expressions and spoken language.

Not only visual perception, but also experience and expression of withdrawal emotions, but not approach emotions, seem to be mediated by the right hemisphere. Positron emission tomography (PET) and electroencephalograph (EEG) studies have revealed that induced negative affective states that are withdrawal-related, as well as production of facial poses of withdrawal emotions, are associated with increased activation in cortical regions in the right hemisphere (Davidson et al., 2000; Coan et al., 2001). Thus, in addition to a right hemispheric specialization in expression and perception of facial withdrawal emotions as well as experience of withdrawal emotions as suggested by these studies, the present data suggest the right hemisphere is crucially involved in prosodic perception of withdrawal emotions.

Interestingly, a trend in decreasing reaction times for semantic perception of (withdrawal) emotions was observed after TMS to the right fronto-parietal operculum. Although this trend effect should be interpreted with caution, it may point to facilitation of semantic processing after RH TMS. Facilitation effects in previous TMS studies have been explained by disinhibition of areas that are connected to the region exposed to TMS or loss of competition between two brain areas after TMS (Walsh et al., 1999). In the present study, the decrease in reaction times for detecting emotion in semantics after TMS to the 
right fronto-parietal operculum, may for example result from loss of competition between this area and the right ventrolateral prefrontal cortex (PFC) that is involved in linguistic aspects of emotion (Hariri et al., 2000). Hence, the ventro-lateral PFC may process semantic information more efficiently. An alternative explanation could be that subjects are automatically screening both semantic and prosodic aspects of spoken language. When processing of prosodic information is disrupted by TMS, subjects may have more processing capacity for analysing semantic information.

An important issue regarding our TMS effects concerns the potential contribution of differences in difficulty between approach and withdrawal emotions. Consistent with the literature, withdrawal emotions were more difficult to detect than approach emotions, which might make them more sensitive to the effects of TMS. However, whereas this higher difficulty of withdrawal emotions applies to both semantic and prosodic tasks, TMS effects were only observed on reaction times in the prosodic task. Moreover, in the sham condition, faster response times were observed in the prosody condition compared to the semantics condition, suggesting that by this index prosody was easier and thus should be less influenced by TMS disruption. Both findings suggest a pattern of TMS effects that is the opposite of what was observed if difficulty is confounded with TMS disruption. With this in mind, it may be important to note that reaction times analyses were limited to correct responses, so any effects of speed-accuracy trade-off were eliminated from the analyses. Although we cannot completely exclude that difficulty might play a role, as this is inherent to the approach-withdrawal dichotomy, our results are consistent with evidence from lesion studies (Mandal et al., 1999; Anderson et al., 2000), which lends credence to our interpretation. The present study shows that right hemisphere TMS selectively interferes with processing of withdrawal emotions, which might imply that approach emotions are less mediated by the $\mathrm{RH}$. This interpretation is consistent with a recent meta-analysis of 106 imaging studies of emotion that revealed a significant difference in the spatial distributions associated with withdrawal and approach emotions (Murphy et al., 2003). Processing of approach emotions was stronger associated with left hemisphere activation compared to the right, supporting the idea that differences in functional neuroanatomy, rather than differences in difficulty level, can explain our results regarding approach and withdrawal emotions.

A related issue is that TMS was applied to the RH only, which is a limitation of the present study. Future studies should incorporate a more complex experimental design including TMS over the right as well as left hemisphere. This design would allow conclusions regarding lateralization of: (i) emotional prosody and emotional semantics; and (ii), withdrawal and approach emotions.

Another concern could be that our effects of TMS on prosodic perception of emotions could be a result of stimulation of the nearby auditory cortex. Although we can not exclude that unintended stimulation of auditory cortex can have affected our findings, the fact that we found specific effects for withdrawal emotions only is not consistent with this explanation. If TMS would have disrupted auditory analysis as a result of stimulation of the auditory cortex, we would have expected uniform TMS effects on prosodic perception across all types of emotions. Besides this argument regarding functional specificity, we would also like to point out that localization of stimulation site using an MRI-guided neuronavigator has been shown to have a high anatomical specificity,(i.e. of $5 \mathrm{~mm}$; Neggers et al., 2004). The targeted area was always above the superior temporal gyrus.

In summary, the present findings indicate that at the level of the somatosensory cortex, evaluation of emotional meaning appears to rely on distinct neural networks that are sensitive to: (i) the communicative channel through which emotional information is presented; and (ii), types of emotions. Our data suggest that at least part of the somatosensory cortex, the right fronto-parietal operculum, might not serve a global function in comprehension of emotional states of others, but appears to be part of a modality-specific neural network. These findings could contribute to our understanding of how socially relevant information is processed at different levels of organization in the brain.

\section{Acknowledgements}

S.v.R. and A.A. were supported by a VernieuwingsImpuls grant (no 016.026.027) from the Netherlands Organization for Scientific Research (NWO). We would like to thank Bas Neggers for help with NeNa.

\section{Abbreviations}

3-D, three-dimensional; BFV, blood flow velocity; fTCD, functional transcranial Doppler ultrasonography; MRI, magnetic resonance imaging; MT, motor threshold; NeNa, neuronavigation; PFC, prefrontal cortex; $\mathrm{RH}$, right hemisphere; ROI, region of interest; TMS, transcranial magnetic stimulation.

\section{References}

Adolphs, R., Damasio, H. \& Tranel, D. (2002) Neural systems for recognition of emotional prosody: a 3-D lesion study. Emotion, 2, 23-51.

Adolphs, R., Damasio, H., Tranel, D., Cooper, G. \& Damasio, A.R. (2000) A role for somatosensory cortices in the visual recognition of emotion as revealed by three-dimensional lesion mapping. J. Neurosci., 20, 26832690.

Aleman, A., Formisano, E., Koppenhagen, H., Hagoort, P., De Haan, E.H. \& Kahn, R.S. (2004) The functional neuroanatomy of metrical stress evaluation of perceived and imagined spoken words. Cereb. Cortex, 15, 221-228.

Anderson, A.K. \& Phelps, E.A. (2000) Perceiving emotion: There's more than meets the eye. Curr. Biol., 10, R551-R554.

Anderson, A.K., Spencer, D.D., Fulbright, R.K. \& Phelps, E.A. (2000) Contribution of the anteromedial temporal lobes to the evaluation of facial emotion. Neuropsychology, 14, 526-536.

Baum, S.R. \& Pell, M.D. (1999) The neural bases of prosody: Insights from lesion studies and neuroimaging. Aphasiology, 13, 581-608.

Blonder, L.X., Bowers, D. \& Heilman, K.M. (1991) The role of the right hemisphere in emotional communication. Brain, 114, 1115-1127.

Borod, J.C., Martin, C.C., Alpert, M., Brozgold, A. \& Welkowitz, J. (1993) Perception of facial emotion in schizophrenic and right brain-damaged patients. J. Nerv. Ment. Dis., 181, 494-502.

Coan, J.A., Allen, J.J. \& Harmon-Jones, E. (2001) Voluntary facial expression and hemispheric asymmetry over the frontal cortex. Psychophysiology, 38, 912-925.

Damasio, A. (1994) Descartes' Error: Emotion, Reason, and the Human Brain. Grosset/Putnam, New York.

Davidson, R.J., Jackson, D.C. \& Kalin, N.H. (2000) Emotion, plasticity, context, and regulation: perspectives from affective neuroscience. Psychol. Bull., 126, 890-909.

Hariri, A.R., Bookheimer, S.Y. \& Mazziotta, J.C. (2000) Modulating emotional responses: effects of a neocortical network on the limbic system. Neuroreport, 11, 43-48.

Harmon-Jones, E. (2003) Clarifying the emotive functions of asymmetrical frontal cortical activity. Psychophysiology, 40, 838-848.

Kosslyn, S.M., Pascual-Leone, A., Felician, O., Camposano, S., Keenan, J.P., Thompson, W.L., Ganis, G., Sukel, K.E. \& Alpert, N.M. (1999) The role of area 17 in visual imagery: convergent evidence from PET and rTMS. Science, 284, 167-170.

Loo, C.K., Taylor, J.L., Gandevia, S.C., McDarmont, B.N., Mitchell, P.B. \& Sachdev, P.S. (2000) Transcranial magnetic stimulation (TMS) in controlled treatment studies: are some 'sham' forms active? Biol. Psychiatry, 47, 325331.

Mandal, M.K., Borod, J.C., Asthana, H.S., Mohanty, A., Mohanty, S. \& Koff, E. (1999) Effects of lesion variables and emotion type on the perception of facial emotion. J. Nerv. Ment. Dis., 187, 603-609. 
Mitchell, R.L., Elliott, R., Barry, M., Cruttenden, A. \& Woodruff, P.W. (2003) The neural response to emotional prosody, as revealed by functional magnetic resonance imaging. Neuropsychologia, 41, 1410-1421.

Mottaghy, F.M., Gangitano, M., Horkan, C., Chen, Y., Pascual-Leone, A. \& Schlaug, G. (2003) Repetitive TMS temporarily alters brain diffusion. Neurology, 60, 1539-1541.

Murphy, F.C., Nimmo-Smith, I. \& Lawrence, A.D. (2003) Functional neuroanatomy of emotions: a meta-analysis. Cogn. Affect. Behav. Neurosci., 3, 207-233.

Neggers, S.F., Langerak, T.R., Schutter, D.J., Mandl, R.C., Ramsey, N.F., Lemmens, P.J. \& Postma, A. (2004) A stereotactic method for image-guided transcranial magnetic stimulation validated with $\mathrm{fMRI}$ and motor-evoked potentials. Neuroimage, 21, 1805-1817.

Oldfield, R. (1971) The assessment and analyses of handedness: the Edinburgh Inventory. Neuropsychologia, 9, 97-113.

Oliveri, M., Romero, L. \& Papagno, C. (2004) Left but not right temporal involvement in opaque idiom comprehension: a repetitive transcranial magnetic stimulation study. J. Cogn. Neurosci., 16, 848-855.

Pourtois, G., Sander, D., Andres, M., Grandjean, D., Reveret, L., Olivier, E. \& Vuilleumier, P. (2004) Dissociable roles of the human somatosensory and superior temporal cortices for processing social face signals. Eur. J. Neurosci., 20, 3507-3515.
Pridmore, S., Fernandes Filho, J.A., Nahas, Z., Liberatos, C. \& George, M.S. (1998) Motor threshold in transcranial magnetic stimulation: a comparison of a neurophysiological method and a visualization of movement method. J. Ect., 14, 25-27.

Robertson, E.M., Theoret, H. \& Pascual-Leone, A. (2003) Studies in cognition: the problems solved and created by transcranial magnetic stimulation. J. Cogn. Neurosci., 15, 948-960.

Sutton, S.K. \& Davidson, R.J. (1997) Prefrontal brain asymmetry: a biological substrate of the behavioral approach and inhibition systems. Psychol. Sci., 8, 204-210.

Vingerhoets, G., Berckmoes, C. \& Stroobant, N. (2003) Cerebral hemodynamics during discrimination of prosodic and semantic emotion in speech studied by transcranial doppler ultrasonography. Neuropsychology, 17, 93-99.

Walsh, V. \& Cowey, A. (2000) Transcranial magnetic stimulation and cognitive neuroscience. Nat. Rev. Neurosci., 1, 73-79.

Walsh, V., Ellison, A., Ashbridge, E. \& Cowey, A. (1999) The role of the parietal cortex in visual attention - hemispheric asymmetries and the effects of learning: a magnetic stimulation study. Neuropsychologia, 37, 245-251.

Walsh, V. \& Pascual-Leone, A. (2003) Transcranial Magnetic Stimulation: a Neurochronometrics of Mind. MIT press, Cambridge. 